\title{
POBREZA Y VULNERABILIDAD: FACTORES DE RIESGO EN EL PROCESO EDUCATIVO
}

\author{
Lilian Inés Castro Durán \\ Universidad de Concepción (Chile) \\ Rufino Cano González \\ Universidad de Valladolid (España)
}

\begin{abstract}
RESUMEN: En este trabajo se pretende analizar la influencia negativa que la pobreza y vulnerabilidad ejercen en las familias y, por ende, en el proceso educativo de los alumnos de una Comuna de la VIII Región del Biobío (Chile). A la vez, interesa conocer y valorar, más en profundidad, la entidad social más influyente en el desarrollo del ser humano -la familia-, como el grupo social primario de mayor importancia y trascendencia. A continuación, se profundiza en el concepto de pobreza con la intención de abordarlo como una realidad presente y específica de América latina para, desde este escenario, situarnos en la provincia del Biobío, y reflexionar sobre los factores más influyentes. Finalmente, haremos mención a la metodología que se ha aplicado para el desarrollo de esta investigación desde una perspectiva cuantitativa y de corte descriptivo mediante encuesta, y, también, a los resultados obtenidos, a través de los cuales se dará cuenta de cómo una serie de condicionantes sociales, entre ellos la pobreza y la vulnerabilidad, perjudican gravemente a las familias que viven en este entorno, socialmente privadas por el desempleo, la escasez de insumos, la insuficiencia de ingresos mínimos, etc. Este contexto, absolutamente desfavorable, perjudica considerablemente la labor docente que es necesario llevar a cabo para educación integral de los niños de la Comuna.
\end{abstract}

PALABRAS CLAVE: Pobreza, vulnerabilidad, familia, educación, escuela.

\section{POVERTY AND VULNERABILITY: RISK FACTORS IN THE EDUCATION OF THE BOROUGH}

ABSTRACT: In this paper we analyze the negative impact that poverty and vulnerability have on families and, therefore, in the educational process of students in the Municipality VIII region of Biobío (Chile). At the same time, we 
are interested to know and appreciate more deeply the most influential social institution in the development of human beings - the family as the primary social group and of greater importance and significance. Then we stop at the concept of poverty with the intent to treat it as a present reality and specific to Latin America, from this stage, go into the province of Punjab, and reflect on the most influential factors. Finally, we mention that we have applied the methodology for the development of this research from a quantitative perspective and cutting descriptive survey, and also the results obtained, through which we will realize how a series of social conditions including poverty and vulnerability, severely damaging to families living in this environment, socially deprived by unemployment, shortages of inputs, inadequate minimum wage, etc. This context, absolutely unfavourable significantly impairs the teaching that it is necessary to carry out education of children of this Commune.

KEYWORDS: Poverty, vulnerability, family, education, school.

Recibido: 15/01/2012

Aceptado: 16/05/2012

\section{INTRODUCCIÓN}

Las variables de pobreza y vulnerabilidad se perciben como factores perjudiciales y lesivos cuando se viven, periódicamente, en una familia. Estas dos variables, difíciles de superar, repercuten ampliamente en la sociedad y forman parte, diariamente, de la realidad de las diversas comunas de la VIII Región.

Dado que el índice de pobreza de estas comunas de la provincia del Biobío es muy alto, con la consecuente vulnerabilidad que esto acarrea, es evidente que nos encontramos ante un panorama desalentador para la inmensa mayoría de los niños y sus respectivas familias. Y, si bien es cierto que hay organismos que están tratando de ayudar a combatir esta lacra, la realidad muestra, hasta el momento, que no se han encontrado los mecanismos necesarios para superar este mal que tanto perjudica a gran parte de la población de este país.

Se considera la familia como grupo social primario. Cuando un individuo nace, su primer acercamiento a la sociedad se produce en el seno familiar. La familia, desde este punto de vista, es considerada una organización de individuos, basada en ciertos rasgos comunes y destinada a trasmitir su cultura. Normalmente, el individuo se encuentra inserto en una familia que, a su vez, influye, muy significativamente, en cada uno de sus miembros. La familia, pues, es considerada como la agencia de socialización primaria; es quien pone los cimientos necesarios para el futuro desarrollo como personas. La familia introduce a sus miembros en la sociedad por medio de las oportunidades que les ofrece. También crea lazos formando parte de los apegos que configuran la estabilidad emocional de cada individuo. Estos apegos sirven como modelo para relacionarnos, tanto con las personas próximas como con el resto de los adultos. 
Las familias son sistemas con múltiples propiedades; propiedades que no pueden hallarse si se descomponen en sus partes puesto que emergen de las relaciones que se establecen constituyendo así la unidad familiar.

El ambiente, por su parte, facilita las herramientas con las que las personas construyen sus relaciones: "Las personas y sus ambientes físicos y sociales, solo pueden comprenderse en su totalidad si tienen en cuenta el contexto de las intrarrelaciones e interrelaciones entre ellos" (Segado 2011: 60). He aquí la importancia que adquieren los ambientes en los que se desarrolla cada individuo, fundamentales a la hora de buscar respuestas a ciertas actitudes de las personas y su interactuar con la sociedad.

Es así como los estudiosos de la psicología evolutiva apoyan las hipótesis que señalan que los niños no solo reciben la influencia de sus padres, sino que, también, pueden influir en ellos. Es dentro de este contexto familiar donde el niño va configurando su sistema de valores mediante procesos de socialización y educación. Según González (2007) y Comellas (2009) la familia proporciona lo que se consideran condiciones óptimas para el desarrollo de la personalidad de los individuos. Además, como señalan estos autores, la familia tiende a trasmitir y guiar a sus hijos influyendo en sus comportamientos, patrones y modelos a seguir. De ahí la importancia que tienen los valores transmitidos por los padres, especialmente en las primeras etapas de vida, donde la imitación y la influencia de modelos es mayor.

La segunda socialización, tal como la concibe Merino y Fuente (2007), corresponde a la escuela que, como se sabe, forma parte del sistema total en el que, normalmente, se desarrolla el individuo. Por tanto, la familia y la escuela, lejos de ser sistemas cerrados, deben abrirse y apoyarse mutuamente. Parafraseando a Von Bertanlanffy (1976), los sistemas son unidades recíprocamente relacionadas. La familia y la escuela forman parte de este sistema con propiedades holísticas e integradoras. Cada uno de estos sistemas debe absorber, plenamente, cada parte de estos ambientes. El mantenimiento del equilibrio de estos sistemas dependerá, en gran medida, de los recursos que disponga cada uno de ellos.

La familia, consecuentemente, se constituye en el pilar fundamental de referencia y de pertenencia de todo ser humano. En ella es donde se establecen vínculos y relaciones afectivas de mayor fortaleza. Desde ella, la toma de decisiones educativas adquiere pleno sentido en tanto que agente socializador y responsable del proceso educativo en su conjunto, puesto que es aquí donde se dan las primeras pautas de socialización, respeto, cuidado y compromisos por parte de los individuos. Aunque todo el contexto familiar socializa, no necesariamente siempre educa (Pérez, 2010); depende, en gran medida, del tipo de valores que se practiquen en la familia porque, por un lado, puede transmitir valores muy positivos y, por otro, absolutamente negativos, como la agresión y la violencia.

Cuando se está frente a contextos sociales desfavorables, ya sea por razones económicas y/o culturales, educar es más difícil; se convierte, ciertamente, en una tarea ardua y compleja debido a la escasa cultura que arropa a estos escolares insertos en contextos familiares muy vulnerables. Se dice que el medio refuerza esta condición. La vida cultural que se respira en estos contextos desfavorecidos 
siempre es escasa y, en muchas ocasiones, nula. Por consiguiente, una de las mayores dificultades o condicionantes sociales que intervienen en nuestra sociedad es, sin duda alguna, la pobreza.

Considerando los datos elaborados por la Comisión Económica para América Latina' (2006), (desde ahora CEPAL), puede comprobarse que el período comprendido entre 2003 y 2006 ha sido el mejor en términos de reducción de la pobreza. Sin embrago, también se señala que el aumento del Producto Interior Bruto de América Latina no ha traído consigo la disminución de dicha pobreza. Como señala Salles (1999), citado por Sanchiz y Gil (2008), los procesos de reajustes macroeconómicos, a partir de la década de los ochenta, derivan en crisis, tanto por los déficit fiscales como por los provocados por la deuda externa, puesto que producen inestabilidad en el empleo, reducción de subservicios urbanos, etc., traduciéndose en una falta de apoyo a dos de los sectores de bienestar social más importantes, como son: la educación y la salud.

La CEPAL, al medir la distribución de los ingresos en los distintos países de América Latina, señala que son estos países quienes muestran mayores desigualdades en el reparto de la riqueza.

La pobreza provoca vulnerabilidad de las personas, porque, en teoría, la pobreza es un problema social y estructural que debe erradicarse para no producir mayores desigualdades sociales.

Uno de los principales objetivos de la política económica y social de América Latina es lograr la reducción de la pobreza. Esta idea fue compartida por quienes tomaron parte de la Cumbre del Milenio, organizada por Naciones Unidas en el año 2000. La meta fue reducir a la mitad la pobreza extrema entre 1990 y 2015. Por su parte, el compromiso de América Latina fue reducir la pobreza extrema hasta el 9\% para el año 2015. Además, se establecieron metas para la solución de otros problemas sociales que son, a la vez, causas y/o consecuencias de la misma, por ejemplo, "la Educación Primaria, el aumento del número de personas con acceso a agua potable, la disminución de la mortalidad infantil, entre otros..." (Navarro, 2005: 12).

Las inequidades sociales, pues, producen vulnerabilidad, pobreza y exclusión como queda de manifiesto en todos los documentos, para América latina, elaborados por la CEPAL en los años 2004 y 2006.

De todas las regiones que conforman el mapa de Chile, se ha considerado, para esta investigación, la VIII Región y, dentro de ella, específicamente la provincia del Biobío. En la siguiente tabla se recogen los índices de pobreza que corresponden a cada una de las comunas de la provincia del Biobío.

1. Comisión Económica para América Latina: Desde ahora CEPAL. La CEPAL es una de las comisiones regionales de las Naciones Unidas. Se instauró para ayudar al desarrollo económico de América Latina y para regularizar las relaciones económicas de los países entre sí y con las otras naciones del mundo. 
Tabla 1. Índice de pobreza comunal. Provincia de Biobío

\begin{tabular}{|l|c|}
\hline Comuna & \% de Pobreza \\
\hline ALTO BIOBÍO & $49,10 \%$ \\
\hline SANTA BÁRBARA & $35,20 \%$ \\
\hline ANTUCO & $34,40 \%$ \\
\hline SAN ROSENDO & $29,30 \%$ \\
\hline NACIMIENTO & $28,50 \%$ \\
\hline NEGRETE & $27,60 \%$ \\
\hline QUILACO & $25,20 \%$ \\
\hline CABRERO & $25,10 \%$ \\
\hline QUILLECO & $24,40 \%$ \\
\hline LOS ÁNGELES & $23,50 \%$ \\
\hline LAJA & $22,40 \%$ \\
\hline YUMBEL & $21,80 \%$ \\
\hline TUCAPEL & $19,80 \%$ \\
\hline MULCHÉN & $19,10 \%$ \\
\hline
\end{tabular}

Fuente: CASEN, 2009.

La tabla 1 indica las comunas con mayores índices de pobreza, entre las que destaca la Comuna de Alto del Biobío, con un 49,10\% de pobreza, y Santa Bárbara con un $35,20 \%$; el resto, como se puede observar, fluctúa entre las nada despreciables cifras del $19,10 \%$ y el $34,40 \%$.

Para paliar estos índices de pobreza que se dan a nivel de País, Región y Comuna, se han destinado programas para las familias más desaventajadas social y económicamente. Es así como, en estos momentos, el sistema de protección social del Gobierno ha ido atenuando la pobreza y beneficiando, aunque muy levemente, a sectores de alta vulnerabilidad social.

Ahora bien, continuando con el tema de vulnerabilidad, debe esclarecerse cómo se concibe en nuestro país este término y cuáles son las condiciones específicas que se han de dar para declarar a una familia o persona "vulnerable". La vulnerabilidad social, en palabras de Perona y Rocchi (2001) es entendida como aquella condición social de riesgo, de dificultad, que inhabilita, de manera inmediata o en el futuro, a los grupos afectados, en la satisfacción de su bienestar -en tanto subsistencia y calidad de vida- en contextos socio históricos y culturalmente determinados.

En el año 2008, el Ministerio de Planificación de Chile definió la vulnerabilidad como aquellas determinadas condiciones que padecen las familias que, ante el riesgo, pueden llegar a empobrecerlas gravemente. Esta vulnerabilidad se asocia a:

- La edad, tamaño, composición, situación familiar, nivel de escolaridad, situación de salud, de empleo e ingresos. 
- Riesgos, crisis familiares, pérdida de empleo o su precarización, enfermedad grave o muerte de un miembro de la familia, discapacidad, abuso de alcohol y/o drogas, entre otros. (MIDEPLAN² 2008).

Para medir la vulnerabilidad se utiliza la Ficha de Protección Social (FPS). Esta herramienta se inspira en una concepción dinámica de la pobreza o vulnerabilidad como un hecho presente o potencial. A continuación, se recogen algunas particularidades de la FPS:

- Es un instrumento que permite identificar a las familias de acuerdo a su nivel de vulnerabilidad.

- Proporciona un puntaje que refleja la vulnerabilidad familiar.

- La información que aporta permite elaborar puntajes especiales para beneficios y programas específicos (salud, educación, vivienda, trabajo, y otros) que se ofrecen a aquellos sectores que los soliciten.

- En la actualidad está vigente el puntaje especial de Carencias Habitacionales para la postulación a programas de vivienda (MIDEPLAN³ 2008).

Según los datos aportados por el MIDEPLAN (2008), la distribución de las familias en riesgo de vulnerabilidad es la siguiente (Tabla 2):

Tabla 2. Distribución de las familias encuestadas con FPS según vulnerabilidad

\begin{tabular}{|l|c|c|c|c|}
\hline Región & Total Familias & $\mathbf{2 0} \%+$ Vulnerable & $\mathbf{4 0} \%+$ Vulnerable & $\mathbf{6 0} \%$ Restante \\
\hline Arica y Parinacota & 31.314 & $58,2 \%$ & $77,9 \%$ & $22,1 \%$ \\
\hline Tarapacá & 44.423 & $47,0 \%$ & $68,2 \%$ & $31,8 \%$ \\
\hline Antofagasta & 58.927 & $41,6 \%$ & $61,4 \%$ & $38,6 \%$ \\
\hline Atacama & 44.651 & $57,4 \%$ & $77.0 \%$ & $23,0 \%$ \\
\hline Coquimbo & 113.692 & $56,6 \%$ & $79,2 \%$ & $20,8 \%$ \\
\hline Valparaíso & 229.356 & $52,7 \%$ & $75,5 \%$ & $24,5 \%$ \\
\hline O'Higgins & 141.205 & $51.2 \%$ & $75,6 \%$ & $24,4 \%$ \\
\hline Maule & 180.159 & $57,3 \%$ & $82,1 \%$ & $17,9 \%$ \\
\hline Biobío & 346.220 & $58,9 \%$ & $80,9 \%$ & $19,1 \%$ \\
\hline La Araucanía & 189.813 & $66,4 \%$ & $86,2 \%$ & $13,8 \%$ \\
\hline Los Ríos & 66.549 & $62,2 \%$ & $84,3 \%$ & $15,7 \%$ \\
\hline Los Lagos & 137.704 & $62,0 \%$ & $83,3 \%$ & $16,7 \%$ \\
\hline Aysén & 21.699 & $52,4 \%$ & $71,1 \%$ & $28,9 \%$ \\
\hline Magallanes & 18.610 & $39,3 \%$ & $62,5 \%$ & $37,5 \%$ \\
\hline Metropolitana & 667.782 & $47,3 \%$ & $69,1 \%$ & $30,9 \%$ \\
\hline País & 2.292 .104 & $54,2 \%$ & $76,2 \%$ & $23,8 \%$ \\
\hline
\end{tabular}

Fuente: MIDEPLAN 2008. (www.cnass.tie.cl. 24/04/2010. 12:19)

2. Power Point (2008). Instrumento para selección de beneficiarios/as de la Reforma Previsional. En MIDEPLAN. Diapo 3. www.cnass.tie.cl 24/04/2010. 12:19.

3. MIDEPLAN: Power Point (2008). Instrumento para selección de beneficiarios/as de la Reforma Previsional. En MIDEPLAN. Diapo 4-8.www.cnass.tie.cl 24/04/20. 
Como puede evidenciarse, en la VIII Región existe un alto porcentaje de familias en situación de vulnerabilidad. Como consecuencia de esta realidad, el Gobierno implementó una serie de programas y leyes, como la Ley de Subvención Escolar Preferencial, que beneficiaba a estudiantes vulnerables. A través de ellos, se evalúa esta condición de acuerdo a los siguientes ítems: $1^{\circ}$. Pertenecer al programa Chile Solidario; $2^{\circ}$. Estar en el tercio más vulnerable según la Ficha de Protección Social; $3^{\circ}$. Ser nacidos de padres o apoderados situados en el tramo A del Fondo Nacional de Salud; 4․․ Utilidades o ingresos familiares bajos; $5^{\circ}$. Nivel escolar; $6^{\circ}$. Edad de los padres o apoderados; $7^{\circ}$. Situación de ruralidad del hogar.

Por tanto, el concepto de vulnerabilidad implica el reconocimiento de dos cuestiones fundamentales: por un lado, establece una relación entre los elementos externos al grupo social con las características socio-económicas y culturales que posee dicho grupo; por otro, introduce el concepto de activo en las poblaciones pobres, mostrando que las mismas poseen recursos y que hacen uso de ellos de forma continua para mejorar su situación o enfrentar situaciones adversas. Obviamente, existen puntos de encuentro entre pobreza, exclusión y vulnerabilidad. De acuerdo con Clichevsky (2002: 12) "la inclusión parcial significa vulnerabilidad y riesgo".

Es en la familia, considerada como la institución más antigua en la historia, como el referente fundamental en el proceso de socialización de todo ser humano, donde se producen los primeros acercamientos del individuo con la sociedad. La familia constituye un elemento primordial en las diversas etapas de la vida repercutiendo, ampliamente, todo lo que nos aportan cada una de las personas.

Este estudio, además de centrarse específicamente en una Comuna con altos índices de pobreza, describe el tipo de familias que se encuentran, los problemas que están viviendo, lo que está sucediendo en las escuelas y cómo repercute los condicionantes familiares de pobreza y vulnerabilidad en la labor educativa.

\section{MéTodo}

El método que se ha seguido en esta investigación de corte no-experimental es el descriptivo. La metodología aplicada para su desarrollo se ha basado en la aplicación de una encuesta a las familias de niños con necesidades educativas especiales, en total, 143 padres y madres. A efectos de esta investigación, se ha considerado el universo de las 12 escuelas de una Comuna de la VIII Región situada en la provincia del Biobío (Chile).

Para dar curso a esta investigación, se parte del siguiente objetivo general:

- Analizar cómo la pobreza y la vulnerabilidad afecta a las familias de una Comuna de la VIII Región de la provincia del Biobío.

Este objetivo general se traduce en los siguientes objetivos específicos:

- Analizar la realidad familiar de esta Comuna y su incidencia en el aprendizaje.

- Conocer la incidencia de la pobreza y vulnerabilidad en la educación de los niños de la Comuna. 
La muestra seleccionada, a partir de la población objeto de investigación, corresponde a 10 escuelas rurales y 2 urbanas; todas ellas calificadas como vulnerables, por cuanto que presentan altos índices de riesgo que varían entre $80,40 \%$ y 97,20\%. De un total de 143 familias de niños con necesidades educativas especiales, se han implicado colaborando con nosotros, 57; es decir, el 40\% de la población de destino.

Como se ha señalado anteriormente, esta investigación se ha servido de 12 escuelas de una Comuna de de la provincia del Biobío. Su población la conforma un conjunto de familias cuyos hijos acuden a los establecimientos de Educación Infantil y de Educación Básica, tanto urbanos como rurales, ubicados en distintos sectores geográficos de la Comuna.

La muestra es no probabilística de tipo intencionada. Los criterios de selección dependieron del acercamiento de los investigadores al contexto y campo de estudio y de los altos índices de vulnerabilidad presentados por las escuelas (entre $80,40 \%$ a $100 \%$ de vulnerabilidad).

El motivo de aplicar una encuesta a las familias (padres y madres) fue obtener datos objetivos y concretos que nos proporcionasen una información verídica, clara y exacta. Para ello, se elabora una propuesta de aplicación sencilla, -la encuesta-, validada y consensuada por tres pares externos, basada en la opinión de los padres de los alumnos con necesidades educativas especiales y compuesta por 28 ítems, además de aquellas preguntas destinadas a recabar información sobre datos demográficos, en los que se plantean una serie de cuestiones que tienen que ver con el sexo, la ocupación de los padres, los ingresos económicos con los que cuentan las familias, sus opiniones en relación con las instituciones escolares y sus niveles de participación y de apoyo. Su estructura se configura en torno a cuatro bloques, a saber:

Bloque I. Características demográficas.

Bloque II. Opiniones generales sobre el Centro educacional.

Bloque III. Opiniones sobre el nivel de participación de las familias.

Bloque IV. Opiniones sobre el nivel de apoyo de las familias.

Para disponer de una base de datos y, asimismo, proceder a analizar la información obtenida a través de la encuesta para padres, se ha utilizado el programa SSPS 17. A partir de aquí, fueron surgiendo una serie de conceptos clave, ideas, argumentos e interpretaciones que sirvieron para sentar las bases de la investigación. Finalmente, se organiza la información sin perder de vista los objetivos.

\section{Resultados}

El proceso estadístico empleado para el logro de los objetivos de la investigación se desarrolló teniendo en cuenta los siguientes aspectos:

- Composición de la muestra respecto de las variables edad, género, ocupación, nivel educacional, ingresos.

- Frecuencias, porcentajes, presentación de gráficos (de barra y circulares) y tablas de contingencias de los cuatro bloques.

Se detalla, a continuación, el análisis descriptivo y comprensivo de la información de cada bloque. 


\section{Bloque I. Características demográficas de la muestra}

Puede decirse que cada familia en activo (el $46 \%$ son padres trabajadores y $38 \%$ son madres trabajadoras; ambos son quienes aportan ingresos a la familia) recibe un sueldo medio inferior a 147.000 pesos, lo que se corresponde, aproximadamente, con unos $240 €$. Además de que estos ingresos son extremadamente bajos, existe más de un $50 \%$ de familias, como se deduce de la muestra manejada, que no alcanza el sueldo mínimo, fijado por el Estado chileno en 193.000 pesos, es decir, menos de $315 €$.

De acuerdo con lo señalado, la mayoría de las familias se encuentran en situación de descorazonadora pobreza, lo que está provocando que todas ellas vivan en un estado de permanente debilitamiento e indigencia desoladora, lo que acarrea una clara y angustiosa carencia de productos alimenticios básicos y de materiales escolares para sus hijos. Por si esto fuera poco, el Sistema de Salud Pública chileno pone la mano en la herida cuando nos alerta diciendo que existen muchas familias incluidas en lo que se ha llamado "grupo de riesgo biopsicosocial" que sobreviven a la penuria y miseria más desafiante por cuanto que sus ingresos "per cápita" son menores o iguales a 30.000 pesos chilenos (unos $46 €$ ).

Por tanto, como se ha señalado más arriba, en ambos grupos, tanto en el de padres como en el de madres, el nivel de ingresos con el que tiene que vivir toda la familia sigue siendo muy bajo, aún si se compara con el salario mínimo obligatorio fijado por la economía chilena (Figura 1).

Nivel de ingresos (\$) del Padre

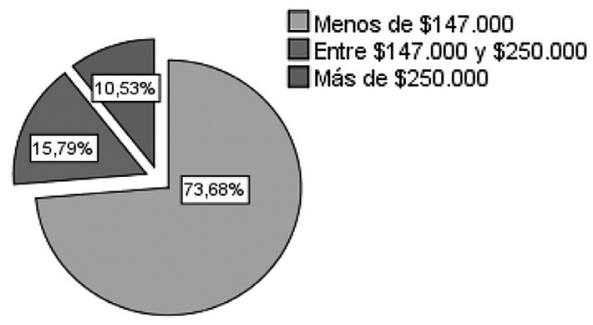

Nivel de ingresos (\$) de la Madre

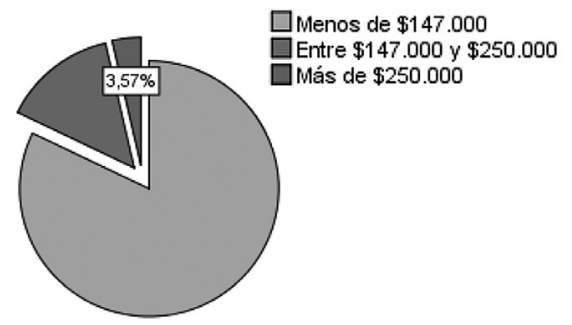

Figura 1. Nivel de ingresos padres (padres-madres)

Tabla 3. Niveles de ingresos padres (padres-madres)

\begin{tabular}{|l|c|c|c|c|}
\hline & \multicolumn{2}{|c|}{ Padre } & \multicolumn{2}{c|}{ Madre } \\
\hline & N & \% & N & $\%$ \\
\hline Menos de 147.000 pesos & 28 & 73,7 & 23 & 82,1 \\
\hline Entre 147.000 y 250.000 pesos & 6 & 15,8 & 4 & 14,3 \\
\hline Más de 250.000 pesos & 4 & 10,5 & 1 & 3,6 \\
\hline Total & 38 & 100,0 & 28 & 100,0 \\
\hline
\end{tabular}

Fuente: Encuesta para padres. 
En relación al nivel de estudios, se constata que el porcentaje más alto se sitúa en el nivel básico, con un 55\% de la muestra total, mientras que el porcentaje "sin estudios" representa el 28,6\%. Con estudios superiores, solamente aparecen 2 mujeres, es decir, el 2,12\% del total de la muestra (Figura 2).

Nivel de estudios del Padre

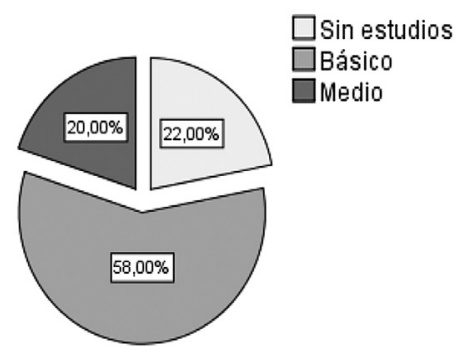

Nivel de estudios de la Madre

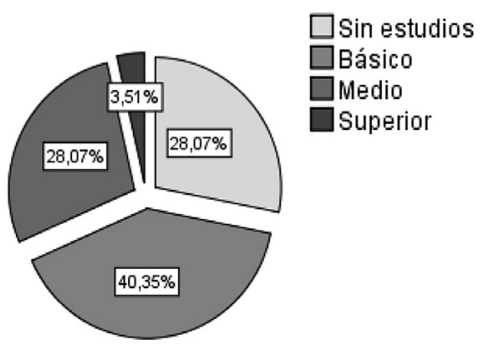

Figura 2. Nivel educativo padres (padres-madres)

A pesar de estos datos, existe un número importante de padres y madres que no certifican estudio alguno; se trata de padres sin escolaridad y/o con unos niveles de escolaridad bajos o muy bajos. En relación con esta cuestión, las investigaciones son muy explícitas señalando que la escolarización es una de las variables o factores de calidad que más influye en las actuaciones educativas de los padres, presentando resultados que avalan que, a menor formación y cultura educativa recibida, más difícil es que los niños obtengan buenos resultados académicos y logren conseguir un desarrollo personal y humano adecuado.

Estos condicionantes, pues, pueden afectar y, de hecho así sucede, a los niños del Ciclo Inicial por una falta de herramientas en los padres para ayudarlos en estos primeros años de vida y de escolaridad obligatoria. Por su parte, los profesores en estos niveles tienen que tener cierto cuidado con los altos índices de repitencia (indicador de deficiencia escolar que se utiliza en Chile para aquellos casos en los cuales los alumnos tienen que repetir más de una vez un grado en los niveles educativos) que afectan a los primeros años escolares, ya que dicha repetición graba negativamente, de una manera muy especial, a aquellos alumnos cuyos padres tienen un nivel formativo y económico bajo o muy bajo. Así lo señalan, entre otros, la UNESCO (1996), Wolff, Schiefelbein y Valenzuela (1994), Halpern (1986) y Jadue (1997), recordándonos la implicación de los padres en el rendimiento de sus hijos por causa de ciertos condicionantes como la pobreza, el nivel educativo y el fracaso escolar, con los que está totalmente relacionado.

A continuación, se hace referencia a las ocupaciones de los padres, destacándose aquellos oficios o actividades que no requieren unos estudios formales previos y que, en este caso, representan una amplia variedad de ocupaciones. En el caso de las madres, se observa que un porcentaje significativo, concretamente el $72,7 \%$, se concentra en la labor o tarea "dueña de casa"; el resto de sus ocupaciones corresponden a oficios que fluctúan entre las frecuencias de 1 a 4 casos (Figura 3). 

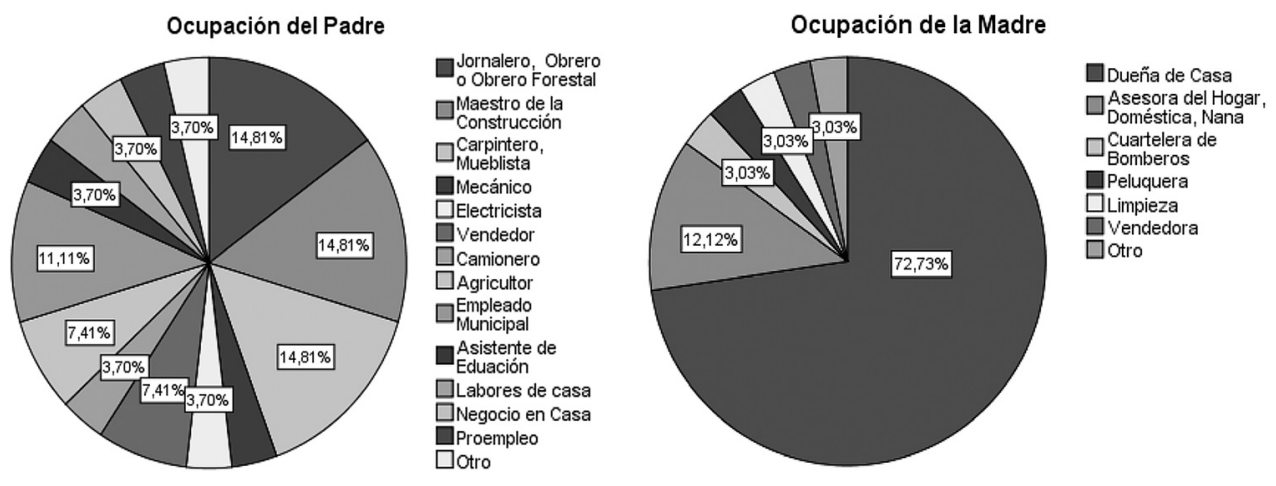

Figura 3. Ocupación de los padres

\section{Bloque II. Opiniones generales sobre el centro educacional}

En este bloque se hace referencia al conocimiento que poseen los padres de los niños con necesidades educativas especiales en relación con el Centro educativo al que acuden sus hijos: metas, reglamentos, instalaciones, admisión e ingreso. Los datos señalan que el $66,1 \%$ de los padres afirma conocer las instalaciones del Centro, mientras que solamente el $51,8 \%$ sabe cuáles son las metas educativas que deben alcanzar sus hijos durante el periodo comprendido entre el inicio y el fin del ciclo escolar en el que se encuentran. Asimismo, existe un 55,6\% de los padres que dicen no conocer los objetivos y metas propios de la escuela que acoge a sus hijos (Figura 4).
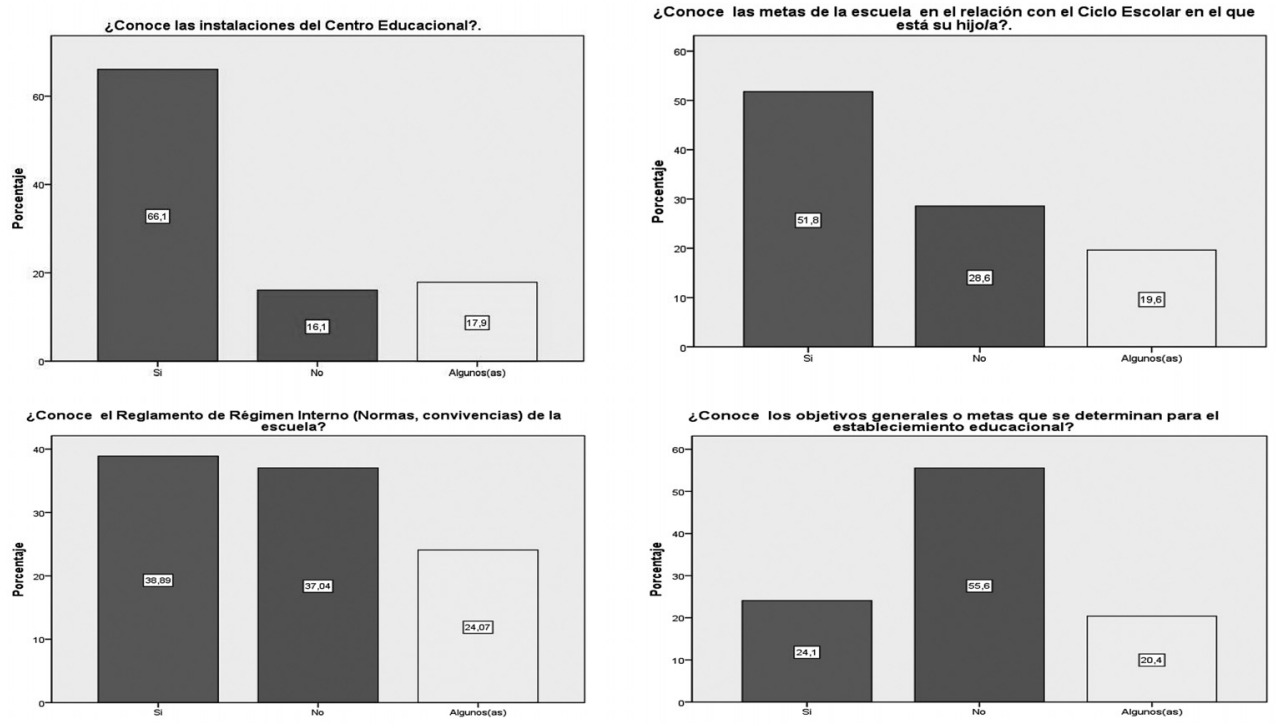

Figura 4. Ítem 1, 2, 3 y 4 
Otro aspecto, igualmente importante y necesario de conocer, es el conocimiento que de sus hijos demuestran tener los padres en relación con sus aprendizajes, sus problemas, sus fortalezas y debilidades. Para ello se acude a la información que proporcionan los ítems 8 y 9, constatando que el 89,5\% de los padres dice conocer los problemas de aprendizaje que tienen sus hijos, mientras que este porcentaje es menor cuando se trata de trasladar dicho conocimiento al ámbito de las debilidades y fortalezas $(69,6 \%)$ (Figura 5).
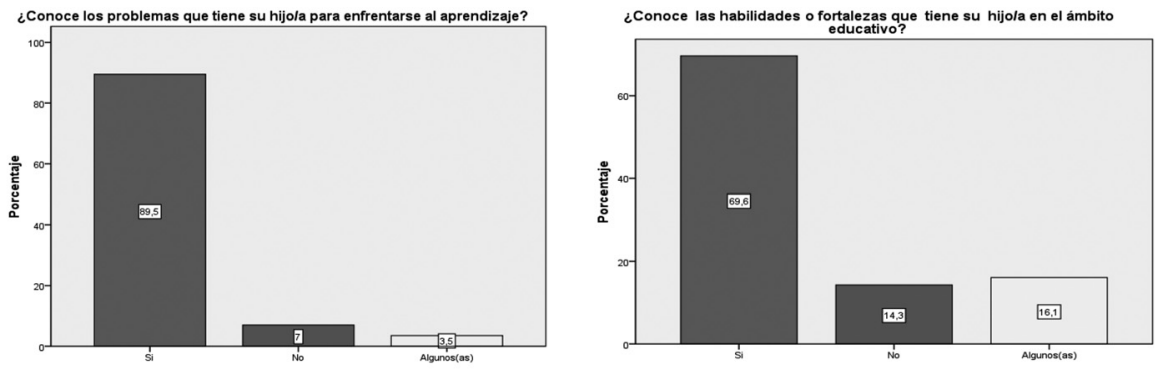

Figura 5. Ítem 8 y 9

Otra variable significativa guarda relación con el grado de conocimiento que poseen los padres acerca de las diversas actividades que se realizan en la escuela. Para ello, primeramente se les pregunta si conocen el Proyecto Educativo Institucional (PEI). Solamente el 32,1\% de los padres afirmó conocer dicho documento institucional, lo que nos llamó poderosamente la atención, puesto que se trata de un documento, -Proyecto Educativo-, en cuya elaboración participan todos los actores educativos, entre ellos, los propios padres. De igual manera ha podido constatarse que el $36,8 \%$ de los padres desconocen las actividades que se programan en el Centro (Figura 6).
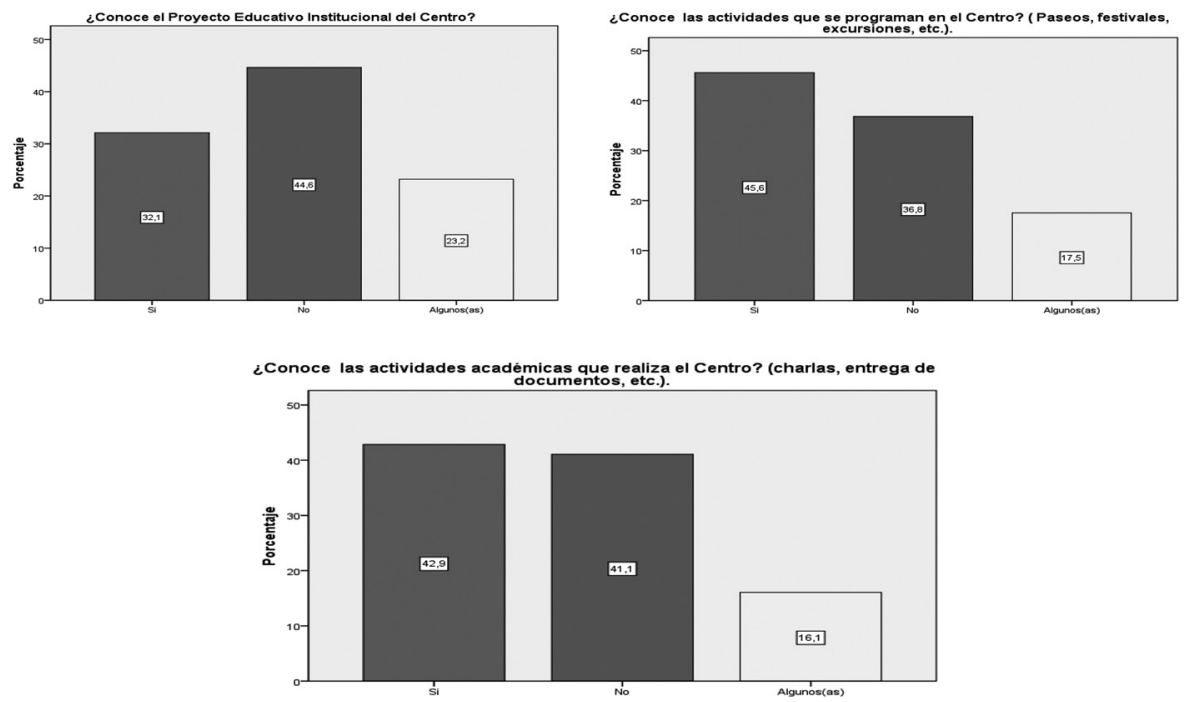

Figura 6. Ítem 10, 11 y 12 
Se concluye señalando que los porcentajes más altos del bloque II, -respuestas afirmativas-, se ubican en los ítems 5, 7 y 8, todos ellos relacionados con el conocimiento que los padres dicen poseer de los responsables de la educación de sus hijos (Director, profesores y profesores de sus hijos), así como de los problemas que tienen sus hijos en relación con el aprendizaje de las diversas materias escolares.

\section{Bloque III. Opiniones generales sobre nivel de participación}

En este bloque se plantea el nivel de participación de los padres en las diversas actividades del Centro: charlas, eventos, reuniones, elaboración de material y otras actividades relevantes que comprometen su participación.

Lo primero que puede constatarse es una baja participación de los padres en el conjunto de las actividades y acontecimientos que, en general, ofrece cada Centro. Aún así, el porcentaje de afirmaciones aumenta, significativamente, en el ítem 8, que mide la participación en cuanto a apoyar procesos de mejora en y del establecimiento, lo que podríamos señalar como positivo (Figura 7).
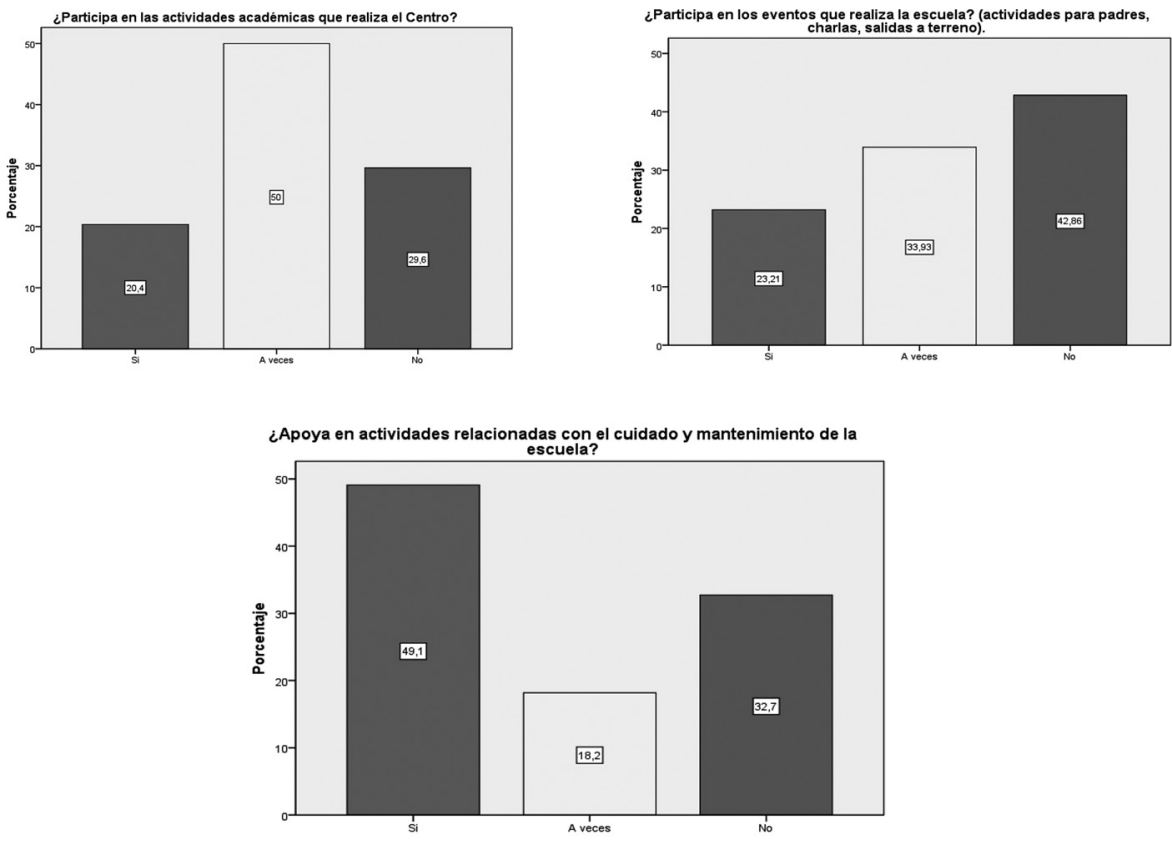

Figura 7. Bloque III. Ítem 1, 7 y 8

En relación con las reuniones y la participación de los padres a nivel de grupocurso, se observa que un $57,4 \%$ de estos asiste, normalmente, a reuniones, y que un $41,07 \%$ colabora en actividades propias del grupo-curso (Figura 8). 

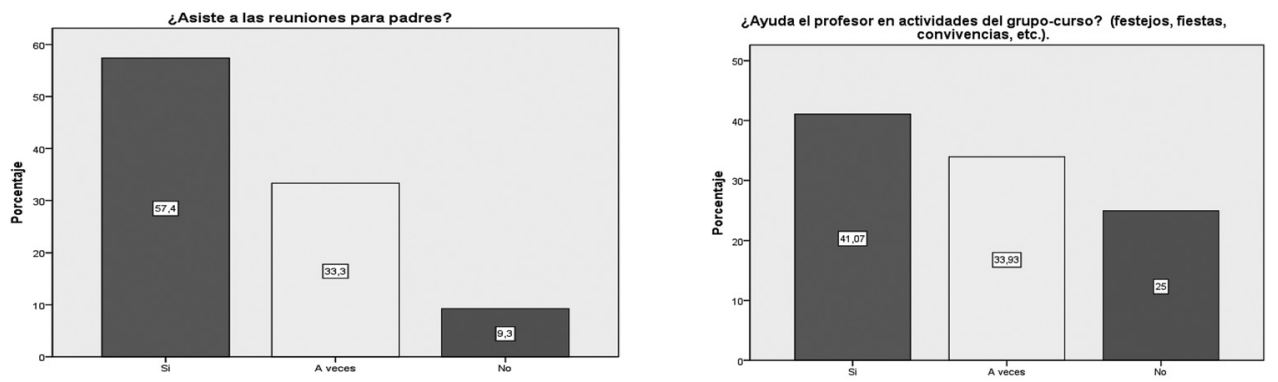

Figura 8. Bloque III. Ítem 2 y 3

Otro ítem es el que se refiere al nivel de ayuda que prestan los padres de cara a la elaboración de material didáctico que el profesor necesita para sus clases. Como se puede comprobar, solamente un 32,73\% de los padres dice ayudar en esta área (Figura 9).

En relación con el acompañamiento que prestan al grupo-curso en actividades fuera del establecimiento, el $47,3 \%$ de los padres señala que no realiza ningún tipo de estas actividades (Figura 9).

Un dato ciertamente interesante es que el $66,1 \%$ conversa con el profesor cuando sus hijos presentan situaciones problemáticas (Figura 9).
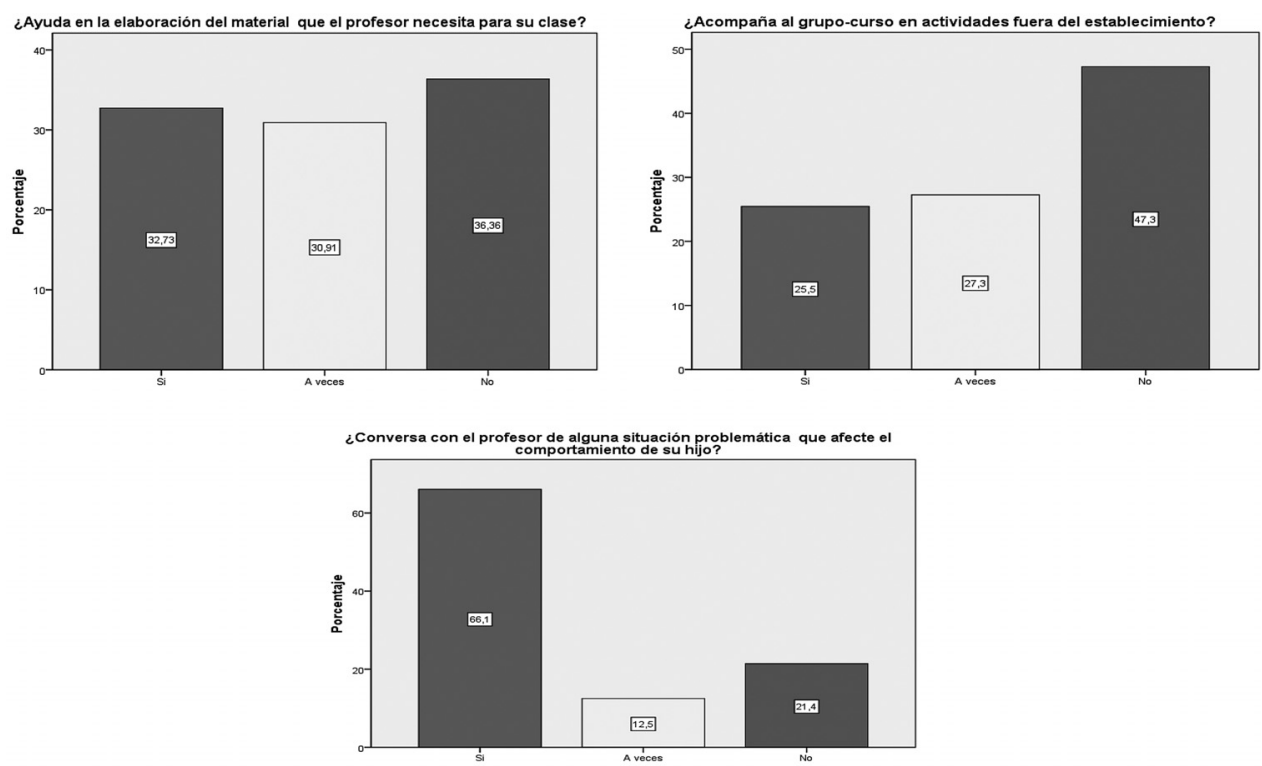

Figura 9. Bloque III. Ítem 4, 5 y 6

Así, pues, se concluye que los ítems del Bloque III más positivos guardan relación con: conversar con el profesor por alguna situación que afecte el comportamiento de sus hijos y asistir a reuniones programadas para padres. 


\section{Bloque IV. Opiniones generales sobre nivel de apoyo}

En este bloque interesa constatar cuál es el nivel de apoyo que los padres prestan a sus hijos en las diversas actividades y labores escolares. Los porcentajes de las respuestas afirmativas son muy altos. Como se puede observar, el $76,4 \%$ de los padres apoyan a sus hijos en las labores de la escuela; el 75\% revisa, habitualmente, las tareas de sus hijos y, finalmente, un $81,1 \%$ dice garantizar espacios apropiados para estudiar, a la vez que manifiestan proporcionar los materiales necesarios para lograr el cumplimiento de dichas tareas (Figura 10).

Asimismo se refleja una actitud positiva, por parte de los padres, hacia labores académicas de sus hijos, lo que, sin duda alguna, es muy alentador para el futuro de estos niños en integración.
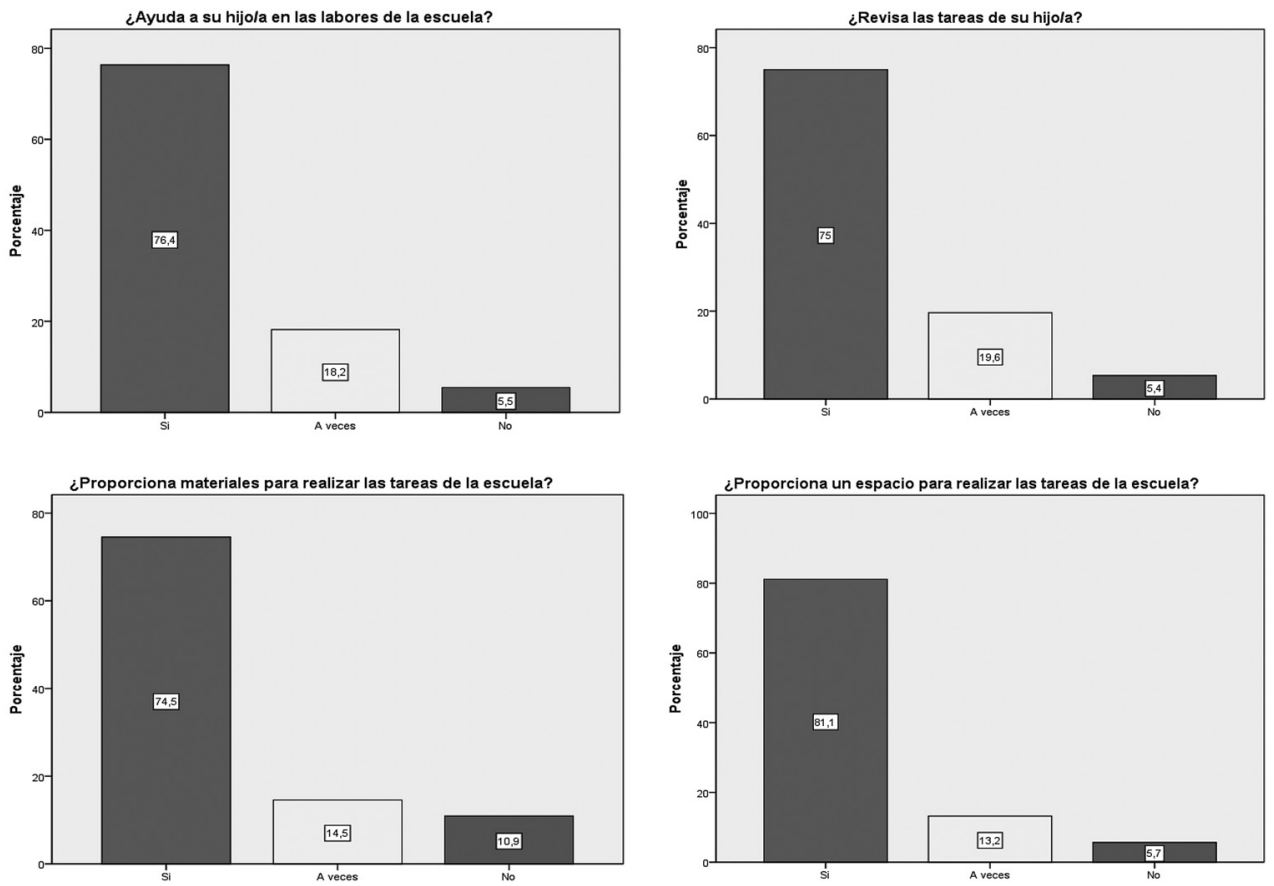

Figura 10. Bloque IV. Ítem 1, 2, 3 y 4

En relación con el apoyo de los padres hacia sus hijos, tanto en actividades de motivación como en aquellas otras interesadas en crear un ambiente adecuado, proporcionar alimentación y estimular la asistencia de sus hijos a la escuela (ítem 5 al 8), los porcentajes correspondientes a las respuestas afirmativas sobrepasan el $80 \%$ (Figura 11). 

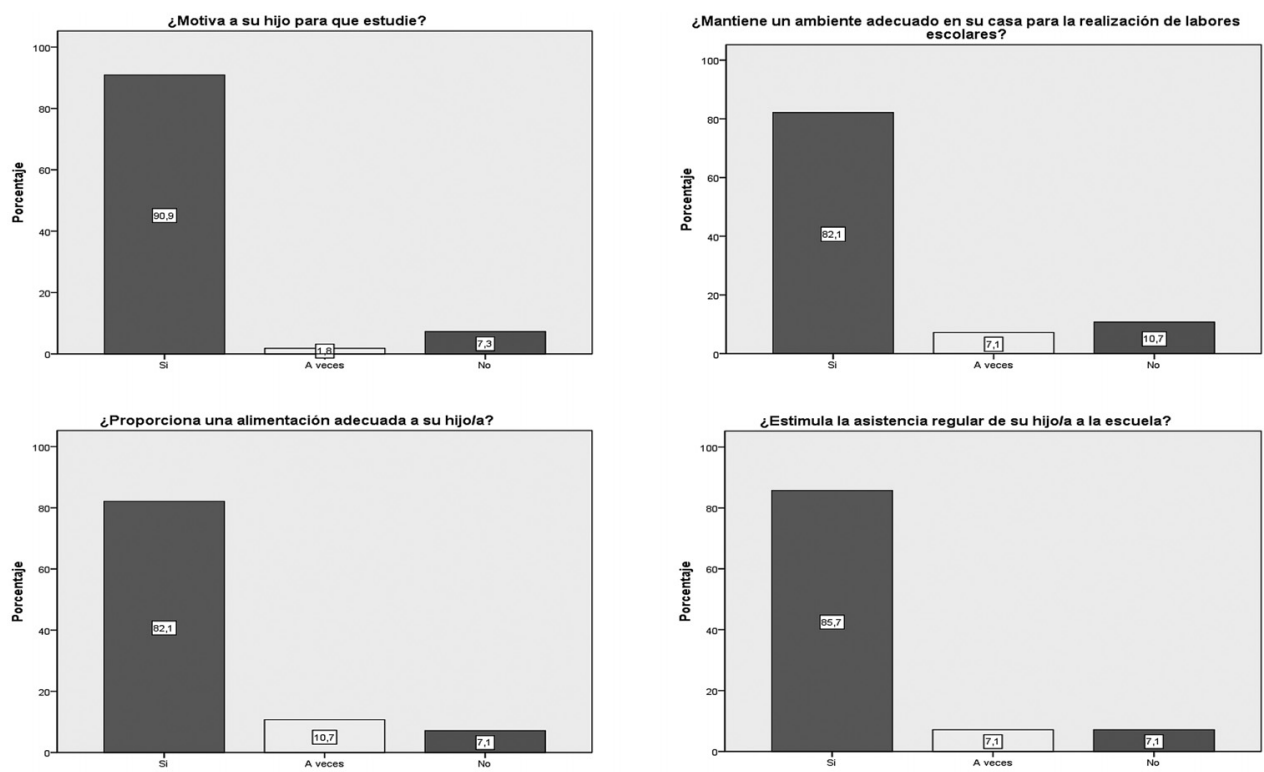

Figura 11. Bloque IV. Ítem 5, 6, 7 y 8

Finalmente, es importante señalar y destacar que de todos los ítems de este bloque IV, el número 5 (Apoyo de los padres a sus hijos en las labores o tareas escolares) es el que obtiene un mayor porcentaje de respuestas positivas, concretamente por encima del $70 \%$.

En resumen, nos encontramos ante un universo de familias marcadas con altos índices de pobreza y vulnerabilidad, como señala Jadue (1991) con una población de niños agredidos por riesgos muy relevantes, fundamentalmente a nivel psicobiológico, social y económico.

Es evidente que las características, absolutamente negativas, que definen a los hogares en permanente situación de riesgo socio-económico, pueden perjudicar, y de hecho perjudican, el aprendizaje de los niños. Factores tales como el ambiente, el clima del hogar, la falta de incentivos para la motivación y la inmersión en el aprendizaje, etc., pueden traer, como consecuencia, que los alumnos no dispongan o no tengan adquiridos aquellos requisitos mínimos exigibles para el aprendizaje de la lectura y escritura presentando, por este motivo, problemas desde el inicio de la etapa escolar. Aunque los padres demuestren apoyo constante a sus hijos y quieran que estos tengan buen rendimiento, sin embargo la mayoría de ellos, como señala Jadue (1997), no utilizan estrategias adecuadas ni disponen de las condiciones ambientales necesarias para mejorar y ayudar a sus hijos.

\section{Conclusiones}

Las situaciones de alto riesgo, como lo son la pobreza, la marginación y la vulnerabilidad, conllevan a enfrentarse a contextos concretos muy difíciles de resolver. 
La importancia que tiene la atención y el apoyo de los padres a sus hijos, es fundamental. De acuerdo con Pérez (2003), el factor entorno es el que más influye en la persona, pero si, además, en ese entorno existen niños con algún tipo de discapacidad, dicho apoyo adquiere tintes de atención doblemente relevante. La familia, pues, se presenta como el núcleo fundamental y prioritario que debe ofrecer los apoyos y recursos necesarios a todos los miembros de su comunidad a lo largo del proceso educativo.

Los padres son los primeros favorecedores y potenciadores del aprendizaje de sus hijos. Es en el hogar donde se construye la base de la educación. El apoyo de la familia es fundamental. La participación activa y el compromiso por parte de los padres generan un proceso educativo y escolar con mayores logros y beneficios.

La mayoría de las familias presentan factores que alteran y perjudican seriamente su labor y responsabilidad educativa como, por ejemplo: su bajo nivel formativo, la falta de fuentes laborales, el cobro de salarios mínimos, los padres cesantes y la pobreza, entre otros. Todos estos indicadores negativos avalan y confirman, al respecto, la teoría de Bronfenbrenner (1987), lo que, sin duda alguna, significa que el contexto socio-económico y cultural afecta, sí o sí, a la forma o manera de relacionarse los individuos entre sí dentro del ámbito educacional.

De lo anterior puede inferirse que existe una alteración importante en los microsistemas escolar y familiar que, como es obvio, está impidiendo un desarrollo normal en los niños que frecuentan este tipo de escuelas cuya imagen se manifiesta, muy especialmente, en el conjunto de déficit académicos y de problemas de aprendizaje que les acompañan permanentemente.

\section{RefERENCIAS BibliogRáficAS}

BRONFENBRENNER, U. (1987). La ecología del desarrollo humano. Barcelona: Paidós. COMELLAS, M. (2009). Familia y escuela: compartir la educación. Barcelona: Graó. COMISIÓN ECONÓMICA PARA AMÉRICA LATINA Y EL CARIBE (1991). Magnitud de la pobreza en América Latina en los años Ochenta. Estudios e Informes de la CEPAL, 81.

COMISIÓN ECONÓMICA PARA AMÉRICA LATINA Y EL CARIBE (2006). Panorama social de América Latina. Naciones Unidas. Disponible en: http://www.eclac.org/ publicaciones/xml/0/27480/PSE_2006.pdf. 24/10/2011.

CLICHEVSKY, N. (2002). Pobreza y políticas urbano-ambientales en Argentina. Serie Medio-ambiente y desarrollo, CEPAL-ECLAC, 49, 1-32.

ENCUESTA de Caracterización Socioeconómica Nacional (Casen) 2009. Documento metodológico. Chile: Gobierno de Chile, Ministerio de Desarrollo Social.

GONZÁLEZ, E. (2007). Aprendizaje y diversidad educativa. Madrid: CCS.

HALPERN, R. (1986). Effects of early childhood intervention on primary schools progress on Latin America. Comparative Education Review, 30, 2.

JADUE, G. (1991). Problemas educacionales que plantean los niños con deprivación sociocultural. Estudios Pedagógicos, 17, 111-123. 
JADUE, G. (1997). Factores ambientales que afectan el rendimiento escolar de los niños provenientes de familias de bajo nivel socioeconómico y cultural. Estudios Pedagógicos, 23, 75-80.

MERINO, R y FUENTE, G. (2007). Sociología para la intervención social y educativa. Madrid: Editorial Complutense.

MIDEPLAN (2008). Instrumento para selección de beneficiarios/as de la Reforma Previsional. Disponible en: www.cnass.tie.cl.

NAVARRO, H. (2005). Manual para la evaluación de impacto de proyectos y programas de lucha contra la pobreza. Serie Manuales, 41. Santiago de Chile: CEPAL.

PERONA, N. y ROCCHI, G. (2001). Vulnerabilidad y Exclusión Social. Una propuesta metodológica para el estudio de las condiciones de vida de los hogares. Cairos, 8. Disponible en: http://www.quadernsdigitals.net/datos_web/hemeroteca/ r_39/nr_408/a_5660/5660.htm.

PÉREZ, P. (2003). Técnicas de intervención en educación especial. Madrid: McGraw Hill.

PÉREZ, P. (2010). Infancia y familia. Valores y estilo de educación (6-14 años). Valencia. Universidad de Valencia.

SANCHIZ, P. y GIL P. (2008). Marginación y Pobreza en América Latina. Estrategias de supervivencias, políticas gubernamentales y acción social. Sevilla: Demos.

SEGADO, S. (2011). Nuevas Tendencias en el trabajo social con familias. Una propuesta para la práctica desde el empowerment. Madrid: Trotta.

UNICEF/UNESCO (1996). La prioridad es la infancia. Cumplimiento de las metas de la cumbre mundial a favor de la infancia. Santiago, Chile.

VON BERTANLANFFY, L. (1976). Teoría General de los sistemas. Fundamentos, desarrollo y aplicaciones. Madrid: Fondo Cultura Económica.

WOLFF, L.; SCHIEFELBEIN, E. y VALENZUELA, J. (1994). Improving the quality of primary education in Latin America and the Caribbean: toward the 21 st century. World Bank discussion paper, 257. 